\title{
Observations of the atmospheric surface layer parameters over a semi arid region during the solar eclipse of August 11th, 1999
}

\author{
Praveena Krishnan, P K Kunhikrishnan*, S Muraleedharan Nair, Sudha \\ Ravindran, Radhika Ramachandran, D B Subrahamanyam and M Venkata Ramana \\ Space Physics Laboratory, Vikram Sarabhai Space Centre, Thiruvananthapuram 695 022, India. \\ *e-mail: pkkrishnan_spl@yahoo.co.in_pk_kunhikrishnan@vssc.org
}

This paper discusses the observations of the Atmospheric Surface Layer (ASL) parameters during the solar eclipse of August 11th, 1999. Intensive surface layer experiments were conducted at Ahmedabad $\left(23^{\circ} 21^{\prime} \mathrm{N}, 72^{\circ} 36^{\prime} \mathrm{E}\right)$, the western part of India, which was close to the totality path. This rare event provided by nature is utilised to document the surface layer effects during the eclipse period using measurements of high frequency fluctuations of temperature, tri-axial wind components as well as mean parameters such as temperature, humidity, wind speed and subsoil temperature. Analysis showed that during the eclipse period, the turbulence parameters were affected leading to the suppression of the turbulence process, the main dynamic process in the atmospheric boundary layer, while the mean parameters showed variations within the natural variability of the observational period. The spectra of the wind components and temperature indicated decrease in spectral power by one order in magnitude during the eclipse period. The rate of dissipation of turbulent kinetic energy is found to decrease by more than one order during the eclipse period. The stability parameter showed a change from unstable to stable condition during the period of eclipse and back to unstable condition by the end of eclipse.

\section{Introduction}

Solar eclipse provides a unique opportunity for studying the response of the atmosphere and the atmospheric phenomena to the sudden cut off and turn on of the sun's radiant energy. Since Atmospheric Surface Layer (ASL) is the region close to the earth's surface, where the mass, energy and momentum transport to other parts of the atmosphere takes place by turbulence process, a change in the radiative heating or cooling will be felt first in the surface layer before it reaches the free atmosphere. However, less effort has been devoted to study the turbulence and spectral characteristics of the Atmospheric Surface Layer (ASL) during solar eclipse. Some of the Atmospheric Boundary layer experiments conducted during eclipses reported drastic changes in mean parameters and fluxes associated with the eclipse event. Antonia et al (1979) examined the velocity and temperature fluctuations during a solar eclipse (October 23rd, 1976) of $80 \%$ totality over Delinquin, Australia to determine how the surface layer responded to the changes in ground heat flux. The Atmospheric Boundary Layer experiments conducted over India during the total solar eclipse of 16th February 1980 (Narasimha et al 1982; Sethuraman 1982) and also during the total solar eclipse of 1995 (Arulraj et al 1998) witnessed a decrease in near surface temperature, wind speed and also the changes in atmospheric stability following the solar eclipse. Eaton et al (1997) provided evidence for the decrease in refractive index structure parameter and diminishing of the turbulence

Keywords. Atmospheric surface layer; solar eclipse; turbulence spectrum; dissipation rate. 
process followed by the development of KelvinHelmholtz waves during the partial eclipse of May 10th, 1994 (94\%) over a desert region at New Mexico, U.S.A. There are also evidences of the pressure perturbations (Anderson et al 1972; Kunhikrishnan and Murthy 1982), the generation of the internal gravity waves (Chimonas and Hines 1970; Liu et al 1998) and oscillations in ASL parameters (Dolas et al 2002) following the total eclipse event.

On August 11th, 1999, a total solar eclipse occurred and traversed the central Indian region when the southwest monsoon was in its active phase. The eclipse almost merged with the sunset period and the active monsoon phase with associated cloud cover softened the expected dramatic changes in the ASL. In the present paper we are reporting the eclipse-induced changes observed in the mean, turbulence parameters and spectra in the atmospheric surface layer during the sudden diminution of solar energy associated with the solar eclipse.

\section{Site and experimental details}

With the objective of studying the changes in the surface layer parameters due to the sudden removal of radiation, field experiments were conducted over a semi arid region, Ahmedabad $\left(23^{\circ} 21^{\prime} \mathrm{N}, 72^{\circ} 36^{\prime} \mathrm{E}\right)$ close to the totality path of the solar eclipse of August 11th, 1999. Figure 1 (courtesy of F. Espenak, 1997, NASA Goddard Space Flight Centre, http://sunearth.gsfc.nasa.gov/eclipse/eclipse .html) shows the footprint of the moon's shadow during the total solar eclipse over the Indian region. The thick shaded area represents the path, and the isopleths outline area with equal maximum coverage of the solar disk. During the solar eclipse, Ahmedabad experienced $99.712 \%$ obscuration. The eclipse at this location started at 16:55:29 hrs, with the maximum phase at 18:00:28 hrs and ended at 18:58:48 hrs. The sunset occurred at 19:25 hrs. All timings are in Indian standard time (IST). A general description of the local circumstances pertaining to this solar eclipse over Indian region is given in Singh (1998).

A $3 \mathrm{~m}$ high slender tower was installed at the experimental site (the ground of Space Application Centre, Ahmedabad) equipped with instruments to measure both mean and turbulence parameters. The experimental site is a land having nearly flat terrain, covered with small grass. The fluctuations in wind, humidity and temperature at $3 \mathrm{~m}$ level were measured by collocated Gill propeller anemometer, capacitor based humidity sensor (humicap) and fast response platinum resistance thermometer respectively. The three-axis Gill propeller anemometer measures orthogonal components of wind velocity (zonal, meridional and vertical) in the range $\pm 30 \mathrm{~ms}^{-1}$ with an accuracy of $0.1 \mathrm{~ms}^{-1}$. The fast response platinum wire thermometer measures temperature in the range from zero to $50^{\circ} \mathrm{C}$ with an accuracy of $0.2 \%$ of the full scale. The capacitor based humidity sensor has an accuracy of $2 \%$ in the range from 0 to $90 \% \mathrm{RH}$ and $3 \%$ accuracy from $90 \%$ to $100 \% \mathrm{RH}$. In addition to the turbulence measurements, slow response measurements of wind, temperature and soil temperature were made using cup anemometer, platinum resistance thermometer and four level soil temperature sensor $(5,10,20 \& 40 \mathrm{~cm})$ respectively. The sampling frequency of fast measurements is $1 \mathrm{~Hz}$, giving 3600 data points in one hour run. The data sampled at $1 \mathrm{~Hz}$ frequency were recorded directly to $\mathrm{PC}$ while one minute averaged data were stored in memory module connected to the system. Prior to the experiment, all the sensors were calibrated and the procedure was repeated several times to check the reproducibility and long term stability of the measuring system.

The experiments were conducted during the period from 4th to 17th August 1999. The sky was partially cloudy or cloudy during most of the days. The mean parameters were measured continuously for these days. The fast measurements were made only for the selected time period during daytime. But round the clock measurements of fast data were made during 10th to 13th August 1999. The air temperature showed a variation in the range $24-30^{\circ} \mathrm{C}$ and relative humidity varied in the range 70-90\% till August 11th, 1999. The air temperature showed an increase from 12th August onwards and relative humidity varied between 40 and $80 \%$. The wind speed was found to be low with a value ranging up to $2 \mathrm{~ms}^{-1}$ and the wind direction indicates the south-westerly flow over land which is characteristic of the south-west monsoon period. The details on the mean and turbulence characteristics during the period of observation are given elsewhere (Praveena and Kunhikrishnan 2002).

\section{Analysis and results}

\subsection{Variation of mean parameters}

The time series of the temperature measured at $3 \mathrm{~m}$ level on eclipse day is shown in figure 2(a). The box in this figure shows the period including solar eclipse and this is further highlighted in figure 2(b). In figure 2(a) the solid curve with vertical bar shows the average value of temperature on days prior to the eclipse day (starting from 4th August to 10th August) and the dashed curve with vertical bars indicate the average value of air temperature after the eclipse day (from 12th August to 17th 


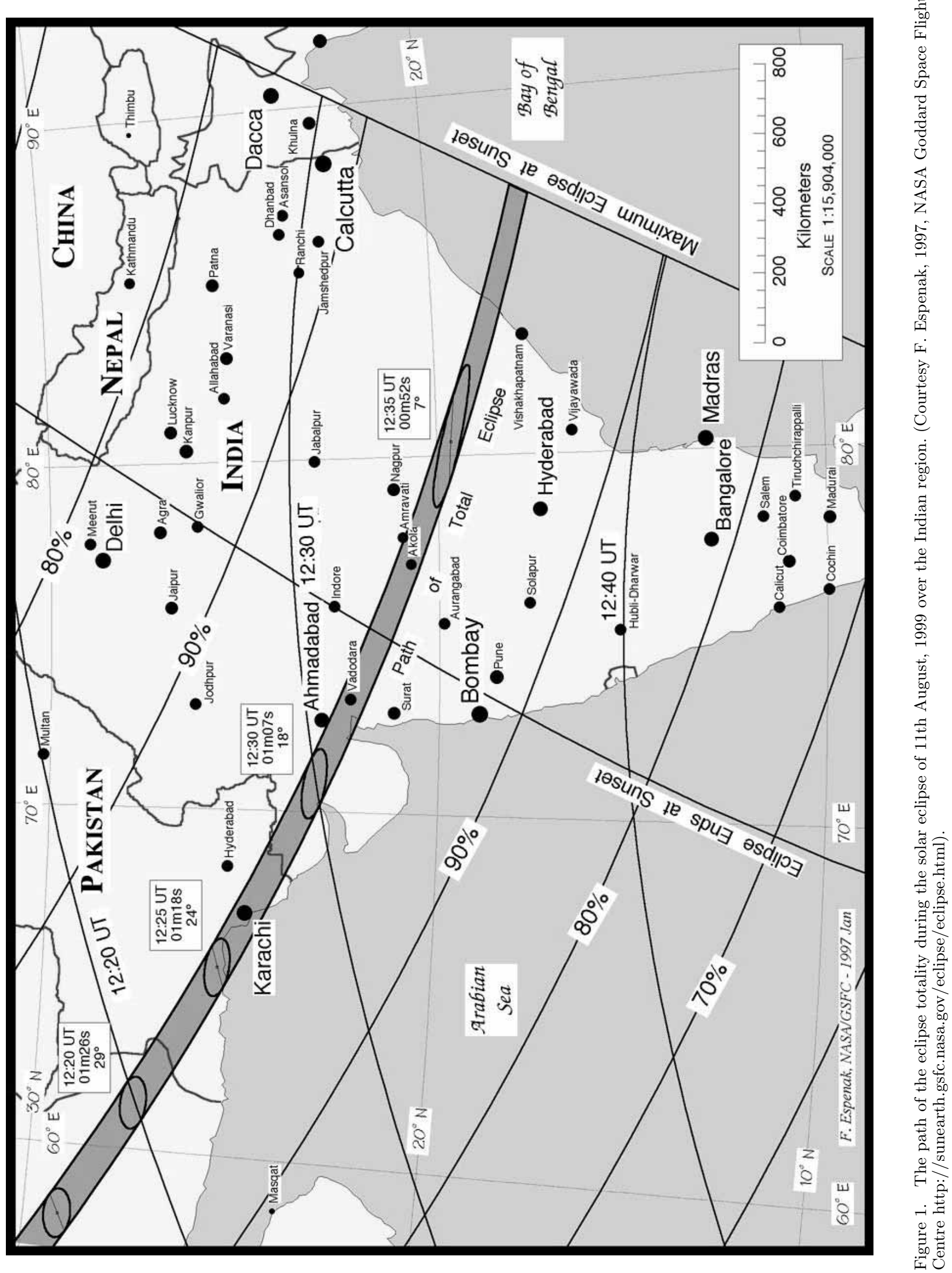



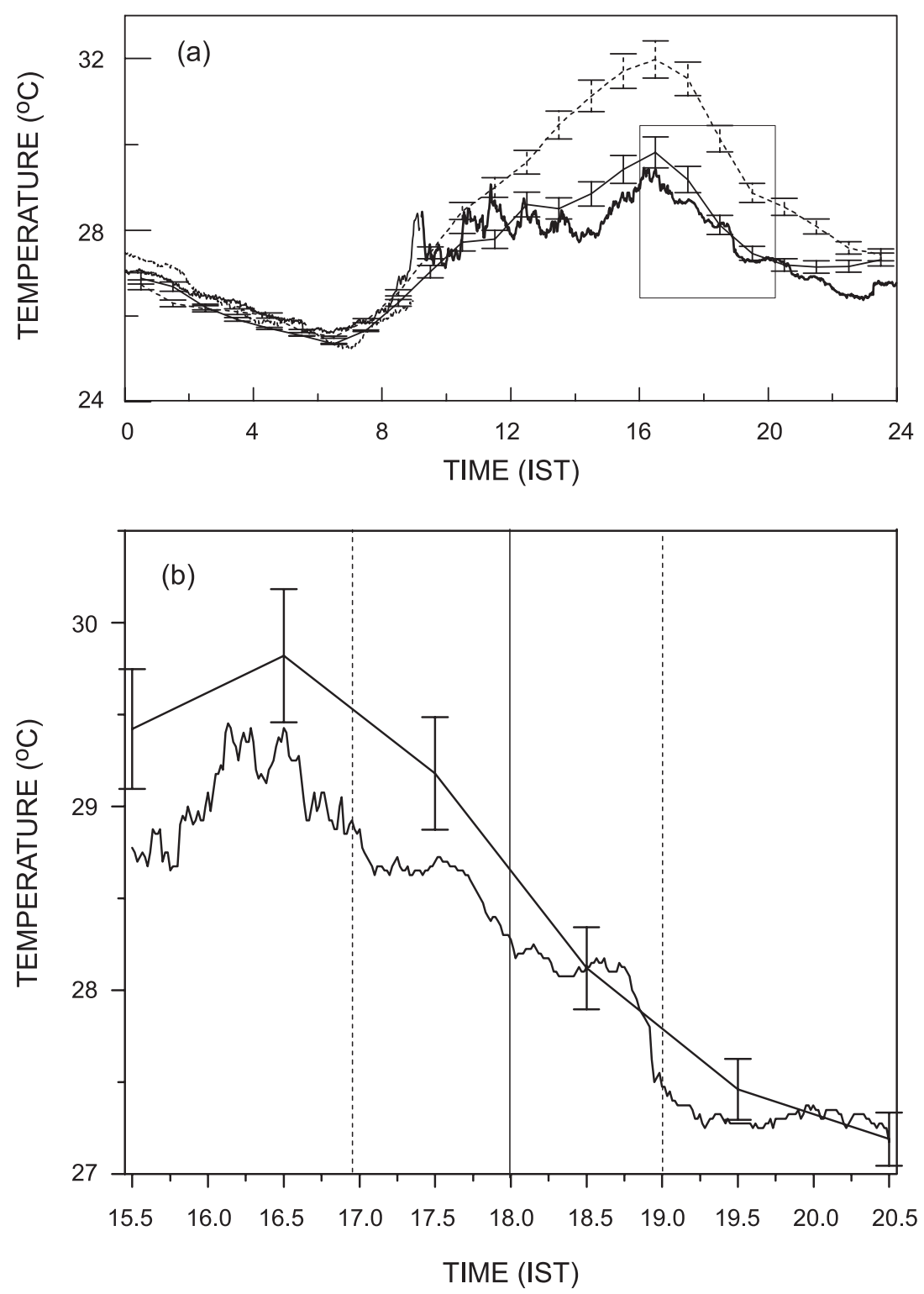

Figure 2. (a) The diurnal variation of temperature at $3 \mathrm{~m}$ level. The solid curve with vertical bars shows the average value of temperature before the eclipse day and the dashed curves with vertical bars indicate the average value of air temperature on days after the eclipse day. (b) Time series of temperature between 1530 and 2030 IST. The onset and end of the solar eclipse are shown with dashed lines; the maximum obscuration time is indicated with solid vertical line. The solid curve with vertical bars shows the average value of temperature prior to the eclipse day. On eclipse day sunset was at 1925 hrs over Ahmedabad.

August). The vertical bars in these figures represent the standard deviation from the mean value. It can be seen that, the mean temperature after the eclipse day (dashed curve) is high (more than $2^{\circ} \mathrm{C}$ ) compared with that on eclipse day and on the days prior to the eclipse days (thin solid curve). This is because of the reduced cloud that prevailed after the eclipse day. So we have compared the mean parameters observed on eclipse day with that observed on days before the eclipse day.

The two dashed lines and straight line parallel to temperature-axis in figure 2(b) shows the onset, end of eclipse and maximum phase respectively. The sunset occurred at 19:25 hrs. The figure shows a decrease up to $\sim 0.50^{\circ} \mathrm{C}$ following the maximum phase of eclipse compared with the mean value and thereafter approaching almost a steady temperature for sometime, and afterwards following a decreasing trend. By the ending phase of the solar eclipse a sudden decrease $\sim 0.80^{\circ} \mathrm{C}$ is visible from the diagram even though the sunset occurred only at $1925 \mathrm{hrs}$. It is observed that air temperature decreased faster following the fourth contact and regained to the mean value during the observational period nearly after one hour. The cut off of solar radiation during the solar eclipse created a condition similar to that in nocturnal boundary layer. This is evident from the stability parameter 
$(z / L)$ in the figure 8(a). It can be seen that surface layer stability became stable in the last phase of eclipse. This can cause radiative cooling in the ground surface. In addition, the radiative divergence within the air can cause cooling in the boundary layer above the ground (Stull 1988). But the present decrease in temperature is not as pronounced as reported by Narasimha et al (1982). They reported a spectacular observation at Raichur during the total solar eclipse of 16th February 1980 , that the temperature started decreasing after the fourth contact. Three hours after the fourth contact, the temperature decreased by $3^{\circ} \mathrm{C}$ than the normal value and they attributed this delayed response to the thermal conductivity of the soil.

The soil temperature observations at four levels below the surface $(5,10,20,40 \mathrm{~cm})$ are shown in figure 3(a). The thick curve represents the soil temperature variations on eclipse day while the thin curve shows the mean value during the noneclipse days prior to the eclipse day. The vertical bars represent the standard deviation from the mean value. It can be noted that the soil temperature at the lower levels $(20,40 \mathrm{~cm})$ are least affected by the eclipse event. The subsoil temperature variation at $5 \mathrm{~cm}$ level nearly follows the air temperature at $3 \mathrm{~m}$ level. But after the ending phase of eclipse, soil temperature cooling is slowed down even though the time rate of change of temperature remains the same as that of the mean value. To see this effect, the profiles of subsoil temperature are shown in figure 3(b). The dotted curve represents the hourly mean profiles for the controlled days (4th to 10th August 1999) whereas half hourly averages are shown for the eclipse day. Thin and thick solid curve represents the first and second half hourly average during the time duration shown in figure 2(b). It can be noted that the soil temperature at $5 \mathrm{~cm}$ depth shows a decrease in temperature by 1730 1800 hours (thick curve) and this is more prominent during 1830-1900 hrs (thick curve) and continued during 1900-1930 hrs in response to the air temperature cooling. Beyond 1930 hrs the soil temperature at $5 \mathrm{~cm}$ shows variation similar to that on controlled days.

Temporal variation of wind speed and relative humidity on the eclipse day and the controlled days are shown in figures 4(a) and 4(b). In these figures the solid curve indicates the mean value observed for the controlled days and the vertical bars show the deviation. Figure 4(a) shows that wind was low during the eclipse period. It can also be noted from figure 4(b) that the relative humidity did not show any appreciable variation during the eclipse period compared to that on non-eclipse days.

\subsection{Turbulence spectra}

Due to the cut off of solar energy during the eclipse event, the thermals cease and the turbulence process is suppressed in the mixed layer with the formation of a residual layer. A better picture of the turbulence process in the surface layer can be represented by turbulence spectra. Spectral analysis of meteorological variables is essentially a statistical method of describing fluctuations, it enables us to study the various aspects of boundary layer structure like the predominant scales of motion, the nature of energy containing eddies, the nature of cascading in the inertial-sub range, the intermittent out break of turbulence induced by terrain and mesoscale processes, modifications by plume and wave activity and the estimation of the size of the eddies that carry maximum energy (Winston et al 1993). So it is interesting to note how the wind and temperature spectra behaved during the total solar eclipse because the spectra indicate the energy transfer process within the boundary layer. For this purpose $1 \mathrm{~Hz}$ data of wind components and temperature were subjected to FFT. It can be noted from figure 2(a) that just before eclipse period itself the temperature shows a decrease around 1200-1300 IST. This may be primarily due to the obscuring by clouds over that region during the period. So it is important to properly identify whether the eclipse induced effects can be seen in the spectra before proceeding to the comparison using other turbulence parameters. With this viewpoint we considered the time period from 1230 to 1930 IST for the spectral analysis.

The ten-minute spectrum for each parameter is found and it is then averaged for one hour to estimate the confidence limits of the spectrum (Jenkins and Watts 1968). So each one-hour spectrum is an average of six ten-minute spectra. Vertical bars shown in figures 5 (a) to (d) represent the $95 \%$ confidence limits of the spectra during 1530-1630 IST period just before the onset of the solar eclipse. Similarly $95 \%$ confidence level is calculated for all spectra. The straight line in each figure represents $-5 / 3$ slope. Figures 5 (a) to (d) depict the spectra of longitudinal $(u)$, lateral $(v)$, vertical $(w)$ components of wind and temperature. It can be seen that the spectra of all wind components are showing nearly one order decrease in power during the maximum phase of the eclipse (1730-1830 hrs) and this effect is more prominent in vertical wind $(w)$ spectra. It can be noted from the figure 2(a) that wind fluctuations are decreased drastically during the period and the slope of spectra remains $-5 / 3$ for all the cases irrespective of the eclipse event. It can also be noted from the figure that the $-5 / 3$ slope of the wind spectra during the maximum phase of the eclipse shifted to the higher frequency 
(a)
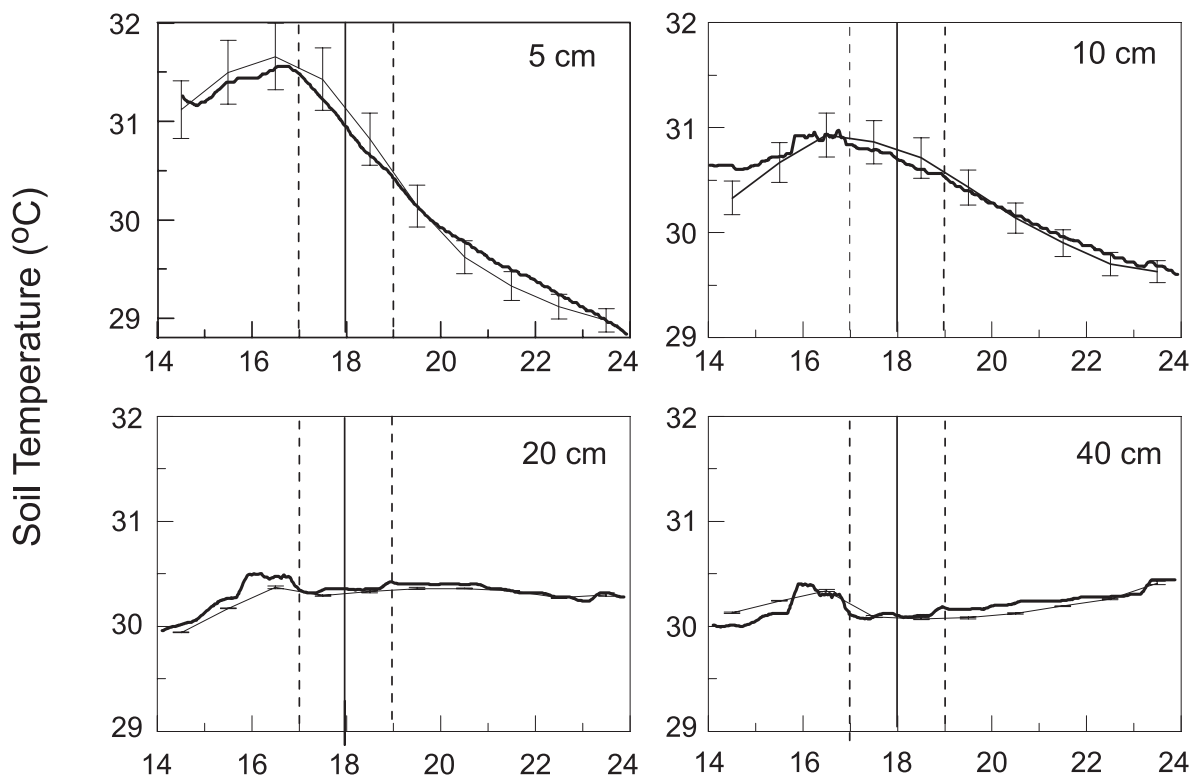

Time (IST)

(b)
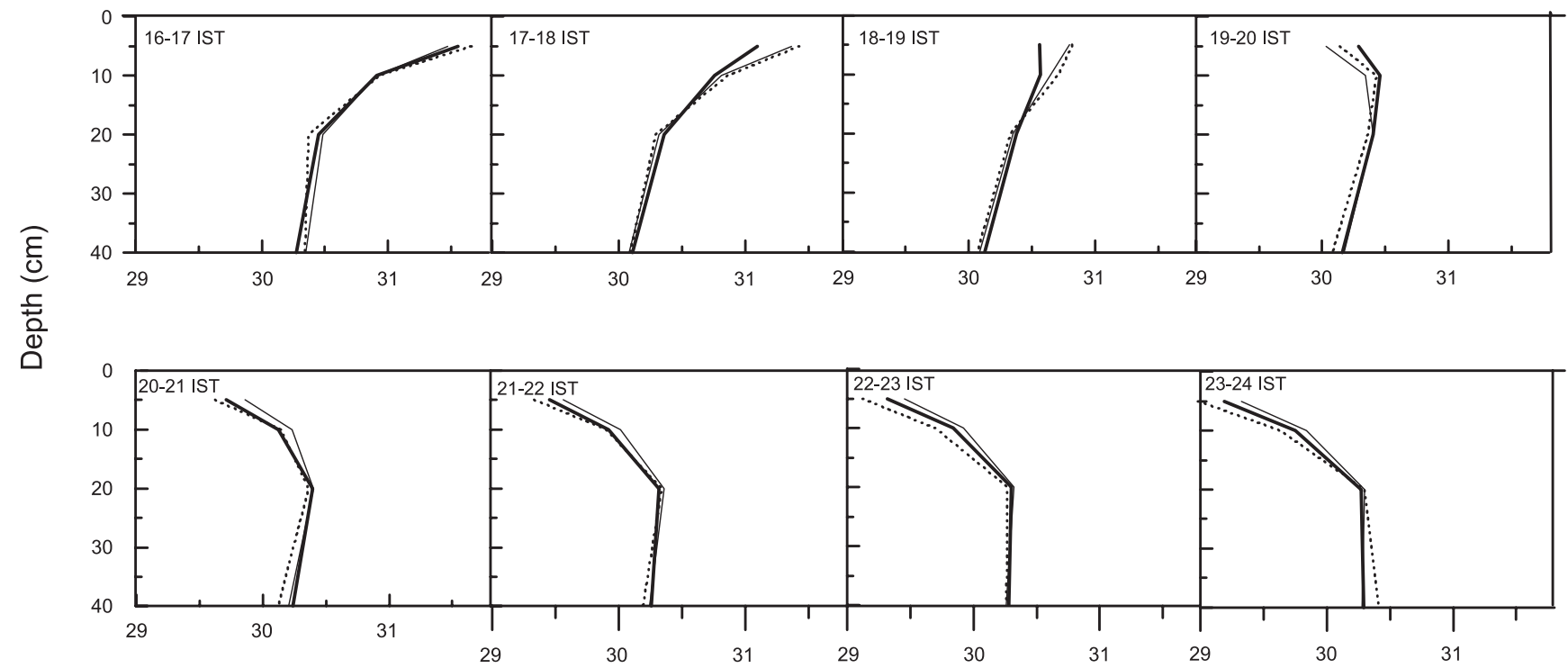

Soil Temperature $\left({ }^{\circ} \mathrm{C}\right)$

Figure 3. (a) Time series of subsoil temperature at different levels. (b) The profiles of subsoil temperature at different depths. The dotted curve represents the hourly mean profiles before the eclipse day (4th to 10th August 1999) whereas half hourly averages are considered for the eclipse day. Thin and thick solid curve represents the first and second half hourly average during the same time duration.

side. This is similar to that observed for a stable boundary layer spectra. It can be seen from figure 8 (a) that the stability parameter $[z / L]$ became positive associated with the maximum phase of eclipse. The peak spectral power of the horizontal wind components is related to the maximum eddy size. Since the boundary layer height is a func- tion of the maximum eddy size (Kaimal and Finnigan 1994), this decrease in eddy size might have resulted in a decrease in boundary layer height. Unfortunately such supporting data was not available during the observational period.

Figure 5(d) shows the temperature spectra observed before, during and after the eclipse. The 

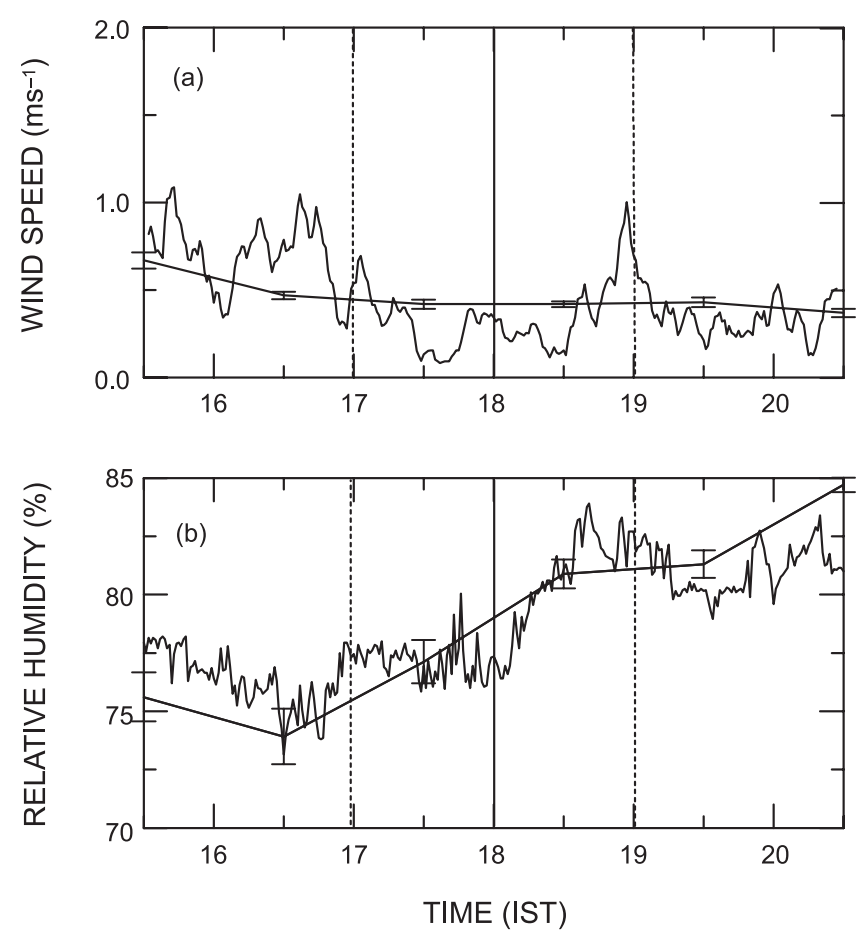

Figure 4. The time series of the (a) wind speed and (b) relative humidity. The solid curve with vertical bars shows the average value before the eclipse day.

temperature spectra also showed a decrease in the spectral power associated with the maximum phase of eclipse. But this decrease in power in the inertial sub range was not as prominent as that seen in wind spectra. Temperature spectra also follow a $-5 / 3$ slope in the inertial sub range. The power level in the temperature spectrum for the duration 1830-1930 hours did not show any increase as seen in the case of wind spectra for the same duration. This can be associated with the decrease in temperature fluctuations as seen in figure 2(b). The change in stability associated with the eclipse is discussed in the section 3.4. Antonia et al (1979) also reported similar spectral behaviour during the solar eclipse (October 23rd, 1976) of $80 \%$ totality over Delinquina, Australia.

\subsection{Dissipation rate}

Since turbulence is driven by a cascade of energy from large scales to small scales, whenever Turbulent Kinetic Energy (TKE) production is large the dissipation rate will also be large. The rate of dissipation of turbulent kinetic energy can be expected to be high if the turbulence process becomes more intense (Stull 1988). This means that there exists a close relation between TKE production rate, intensity of turbulence and dissipation rate. Intensity of turbulence can be defined by the rate at which turbulent kinetic energy is transferred before its final dissipation into heat and it can be parame- terised by examining those scales of motion in equilibrium regime where energy is neither created nor destroyed but passes from larger to smaller scales. So rate of dissipation provides a measure of how much kinetic energy is cascading through equilibrium range. The rate of dissipation of turbulent kinetic energy has been estimated (Caughey and Palmer 1979; Caughey and Wyngaard 1979; Kaimal and Finnigan 1994) from the inertial subrange level of vertical velocity spectrum using Kolmogorov's law (Taylor's hypothesis of frozen turbulence has been used to convert wave numbers to frequencies)

$$
S(f)=\alpha\left(\frac{\bar{u}}{2 \pi}\right)^{2 / 3} \varepsilon^{2 / 3} f^{-5 / 3},
$$

where $S(f)$ is the spectral density, $\bar{u}$ mean velocity, $f$ frequency, $\varepsilon$ the energy dissipation rate, representing the rate of dissipation of turbulent kinetic energy to heat by viscous forces and $\alpha$ the Kolmogorov's constant. For estimating the dissipation rate $\varepsilon$, the universal constant $\alpha$ in the Kolmogorov's expression for one dimensional wind spectrum, is taken as 0.55 (Kaimal and Finnigan 1994). The time evolution of the rate of dissipation of turbulent kinetic energy on eclipse day (solid curve) and the mean value (on other non-eclipse days i.e., 10th, 12th and 13th August 1999) during the experimental period (dotted curve) are shown in figure 6 . The vertical bars show the standard deviation from the mean value. From figure 6 , it is evident that there is a decrease of more than one order in the value of the rate of dissipation of turbulent kinetic energy, associated with the eclipse phase and after maximum eclipse the values attained the mean level during the observational period. The average rate of dissipation of the turbulent kinetic energy for the controlled days considered is found to be of the order of $10^{-2}$ to $10^{-3} \mathrm{~m}^{-2} \mathrm{~s}^{-3}$ while the solar eclipse period experienced a dissipation rate of nearly $10^{-5} \mathrm{~m}^{-2} \mathrm{~s}^{-3}$.

\subsection{Turbulence parameters}

To see the eclipse effects on the turbulence parameters, friction velocity $(u *)$, turbulent kinetic energy per unit mass (TKE), stability parameter $(z / L)$, and fluxes of sensible $\left(Q_{H}\right)$ and latent $\left(Q_{E}\right)$ heat are estimated (Stull 1988; Kaimal and Finnigan 1994) utilizing fast sampled data as

$$
\begin{aligned}
u_{*} & =\left(\overline{u^{\prime} w^{\prime}}+{\overline{v^{\prime} w^{\prime}}}^{2}\right)^{1 / 4}, \\
\mathrm{TKE} & =\frac{1}{2}\left(u^{\prime 2}+v^{\prime 2}+w^{\prime 2}\right),
\end{aligned}
$$


(a)
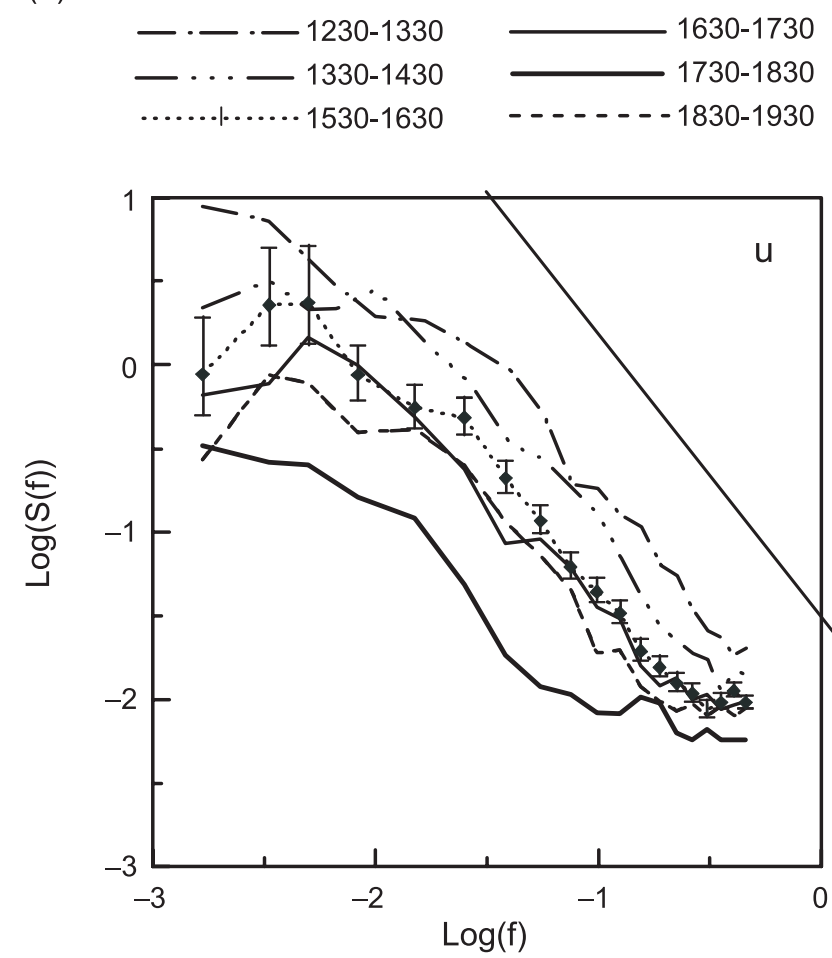

(c)
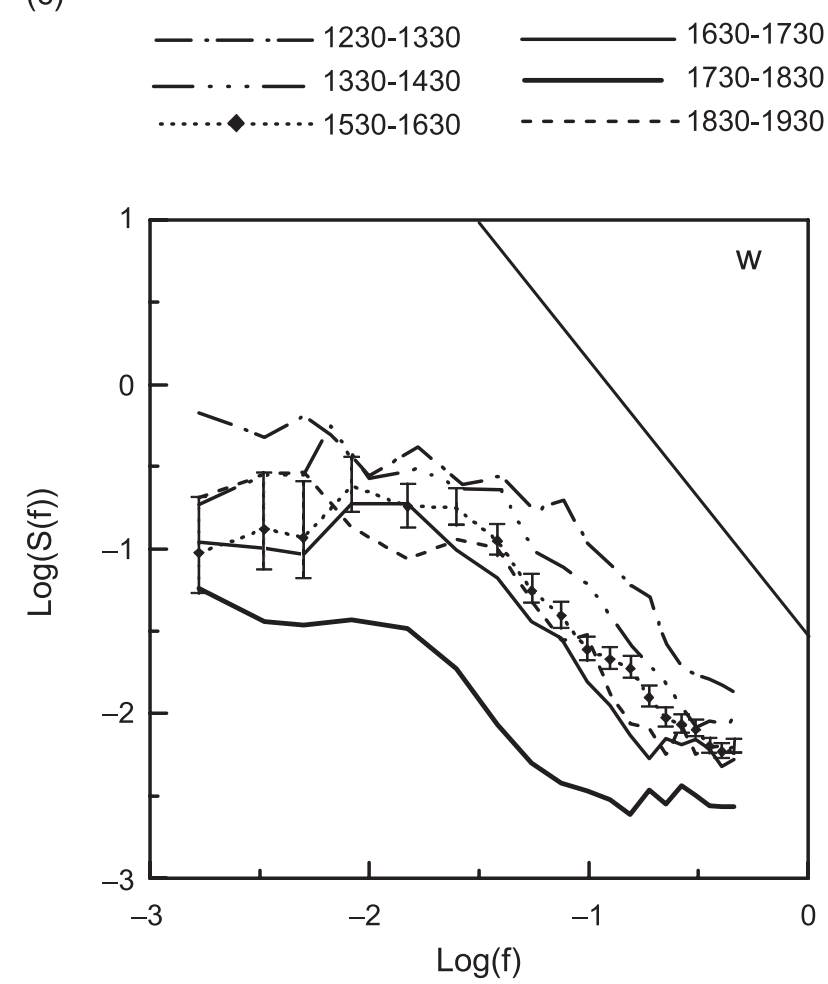

(b)
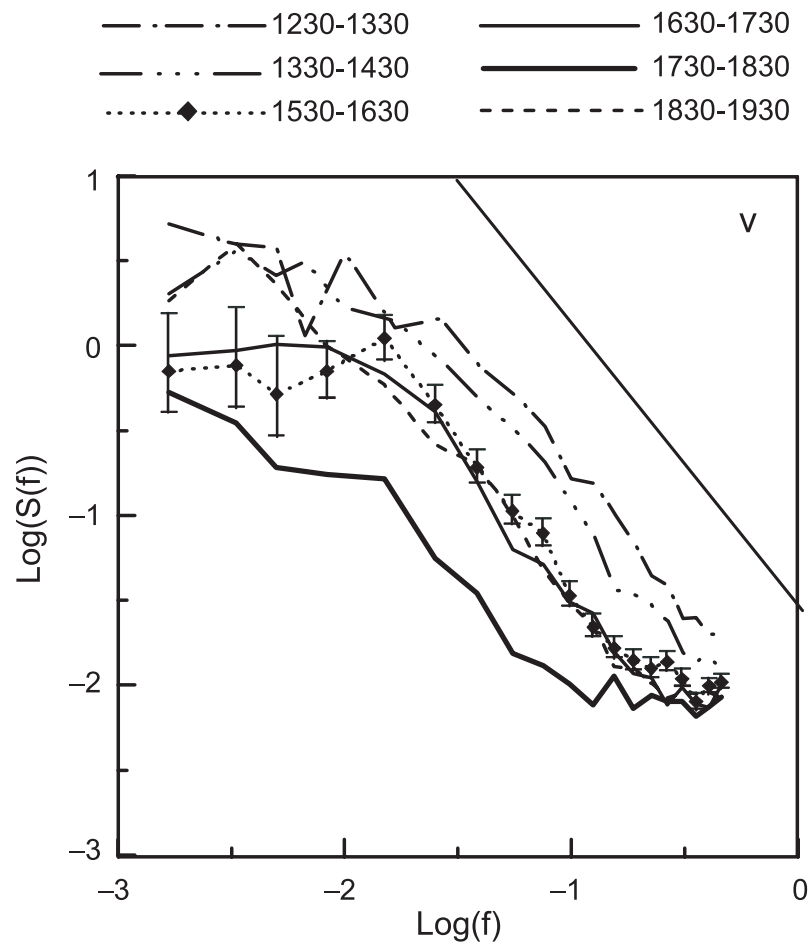

(d)
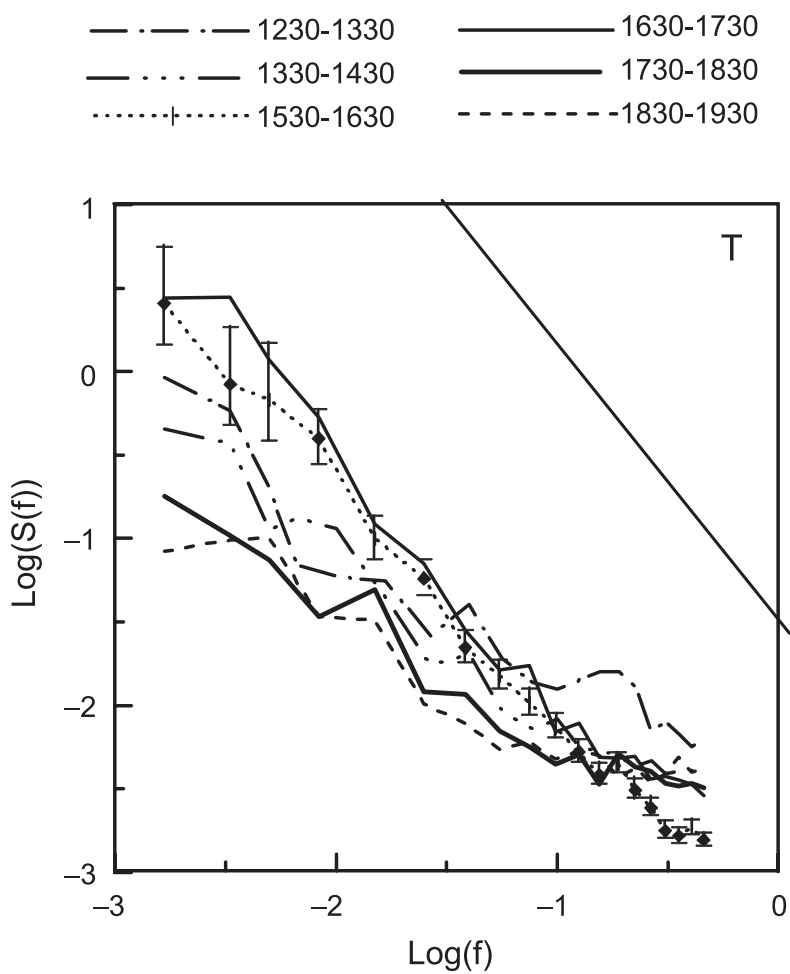

Figure 5. Spectra of (a) $u$, (b) $v$, (c) $w$ and $T$ component on August 11th 1999 during the periods: 1230-1330 IST, 1330-1430 IST, 1530-1630 IST, 1630-1730 IST, 1730-1830 IST and 1830-1930 IST. The solid straight line indicates $-5 / 3$ slope. The vertical bars during 1530-1630 IST show the 95\% confidence limits of the spectra during that period.

$$
\begin{aligned}
\frac{z}{L} & =\frac{-z g k \overline{w^{\prime} T^{\prime}}}{u_{*}^{3} T}, \\
Q_{H} & =\rho C_{p} \overline{w^{\prime} T^{\prime}},
\end{aligned}
$$

$$
Q_{E}=\rho L_{v} \overline{w^{\prime} q^{\prime}}
$$

where $u^{\prime}, v^{\prime}, w^{\prime}$ and $T^{\prime}$ denote the fluctuations in longitudinal, lateral and vertical wind speed 


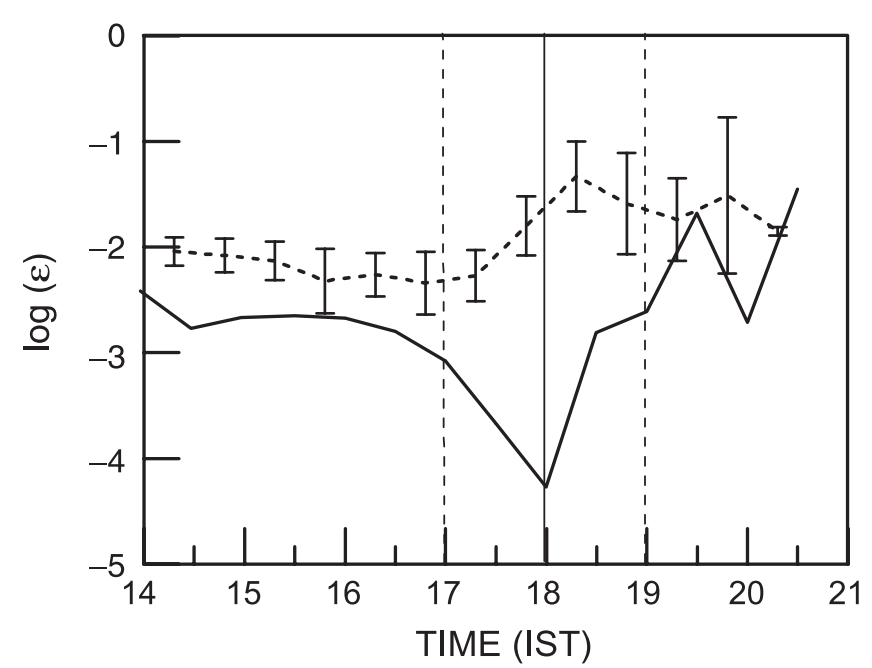

Figure 6. Time series of the variance of rate of dissipation of turbulence kinetic energy at $3 \mathrm{~m}$ level. The onset and end of the solar eclipse are shown with dashed lines; the maximum obscuration time is indicated with solid vertical line. The dashed curve represents the mean value for 10th, 12th and 13th August 1999.
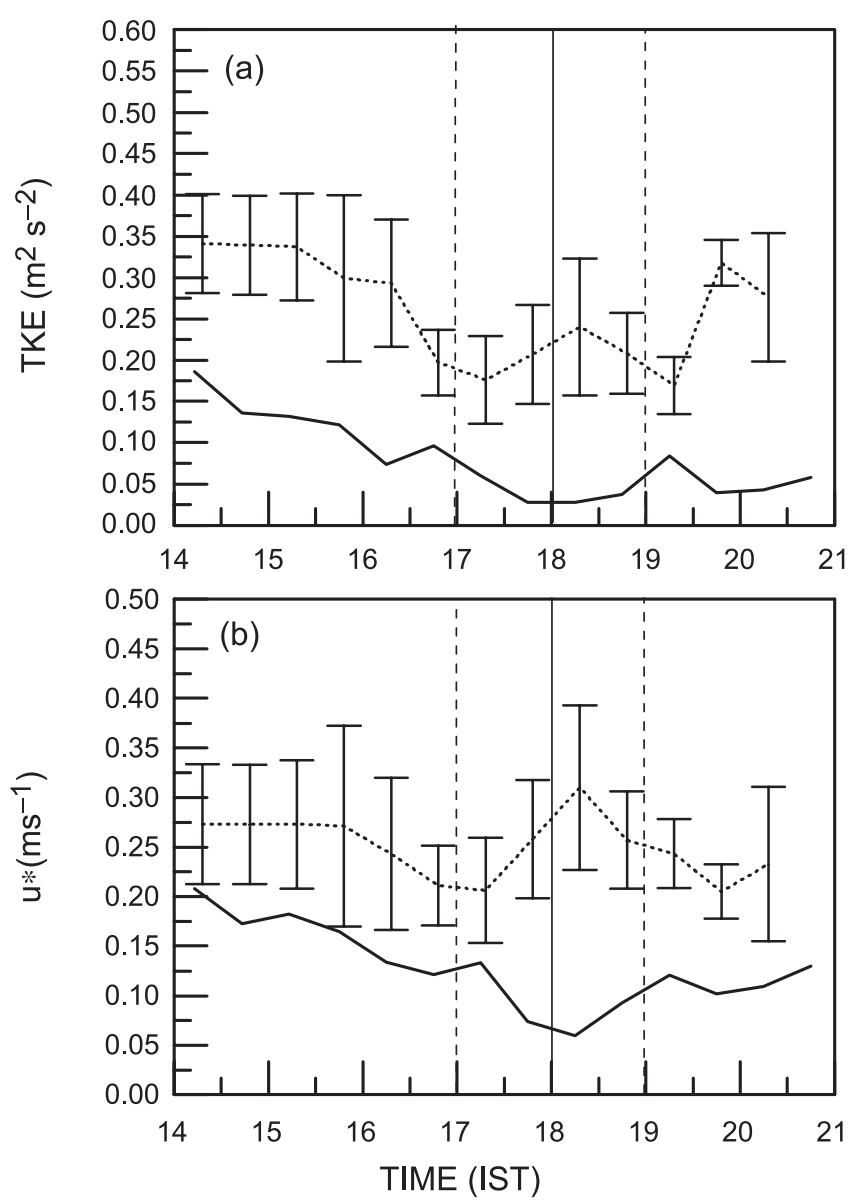

Figure 7. Same as in figure 6 but for (a) turbulent kinetic energy and (b) friction velocity.

respectively. $g$ is acceleration due to gravity and $k$ is Von Karman constant (0.40). $z$ is the height of observation (here, $3 \mathrm{~m}$ ) and $L$ is the Obukhov length. $\rho, C_{p}$ and $L_{v}$ represent the density of air, specific heat capacity of air at constant pressure and latent heat of vaporization respectively.

The 30-minute averaged values of turbulence parameters on eclipse day are shown by solid curve and the non-eclipse days by the dashed curve. The dashed curve with vertical bars represents the mean value for three days (10th, 12th and 13th August, 1999) and the vertical bars show the standard deviation from the mean value. The variation of turbulence kinetic energy and friction velocity are shown in figure 7 . There is a marked decrease in the value of friction velocity and turbulence kinetic energy during the eclipse period as seen from figure 7 . The TKE during the eclipse period reduced to $0.025 \mathrm{~m}^{2} \mathrm{~s}^{-2}$, while friction velocity decreased to $\sim 0.05 \mathrm{~ms}^{-1}$. This change can be attributed to the decrease in turbulence fluctuations of wind components during solar eclipse.

To investigate changes in stability if any, the time evolution of stability parameter is shown in figure $8(\mathrm{a})$. The stability parameter basically expresses the efficacy of buoyancy and wind shear in producing turbulence. The negative of $z / L$ represents unstable conditions, whereas positive values indicate stable conditions. It can be seen that near neutral conditions prevailed during the noneclipse days (figure 8a). On eclipse day the stability condition was nearly unstable before the eclipse. However, the conditions become stable just before the first contact and the transformation from stable to unstable conditions and back to stable condition occurred during the maximum phase of eclipse. The $z / L$ values show unstable condition after the ending phase of the eclipse and following the sunset it again approached the stable value. The overall change in the value of $z / L$ for this day is between -0.15 and 0.10 . The stabilization following the total phase is similar to that reported earlier (Eaton et al 1997; Sethuraman 1982). The solid and dotted curves have the same relevance as explained above. The time evolution of the sensible (figure 8b) and latent heat (figure 8c) shows a decrease by the onset of eclipse followed by an increase just after the last phase of the eclipse. The maximum value of sensible heat flux on eclipse day was only $\sim 40 \mathrm{Wm}^{-2}$ (at $1230 \mathrm{hrs}$ ). It can be seen that the fluxes of sensible and latent are approached values close to zero and negative values following maximum phase of the eclipse. This is almost similar to that which occurs during the nighttime condition. It is a clear indication of the decrease of buoyancy effects. However, it should be noted that the values are not decreasing drastically as reported earlier by Eaton et al (1997) and Arulraj et al (1998), since the eclipse occurred during the evening hours and a partial cloudiness period prevailed during the observational period. 

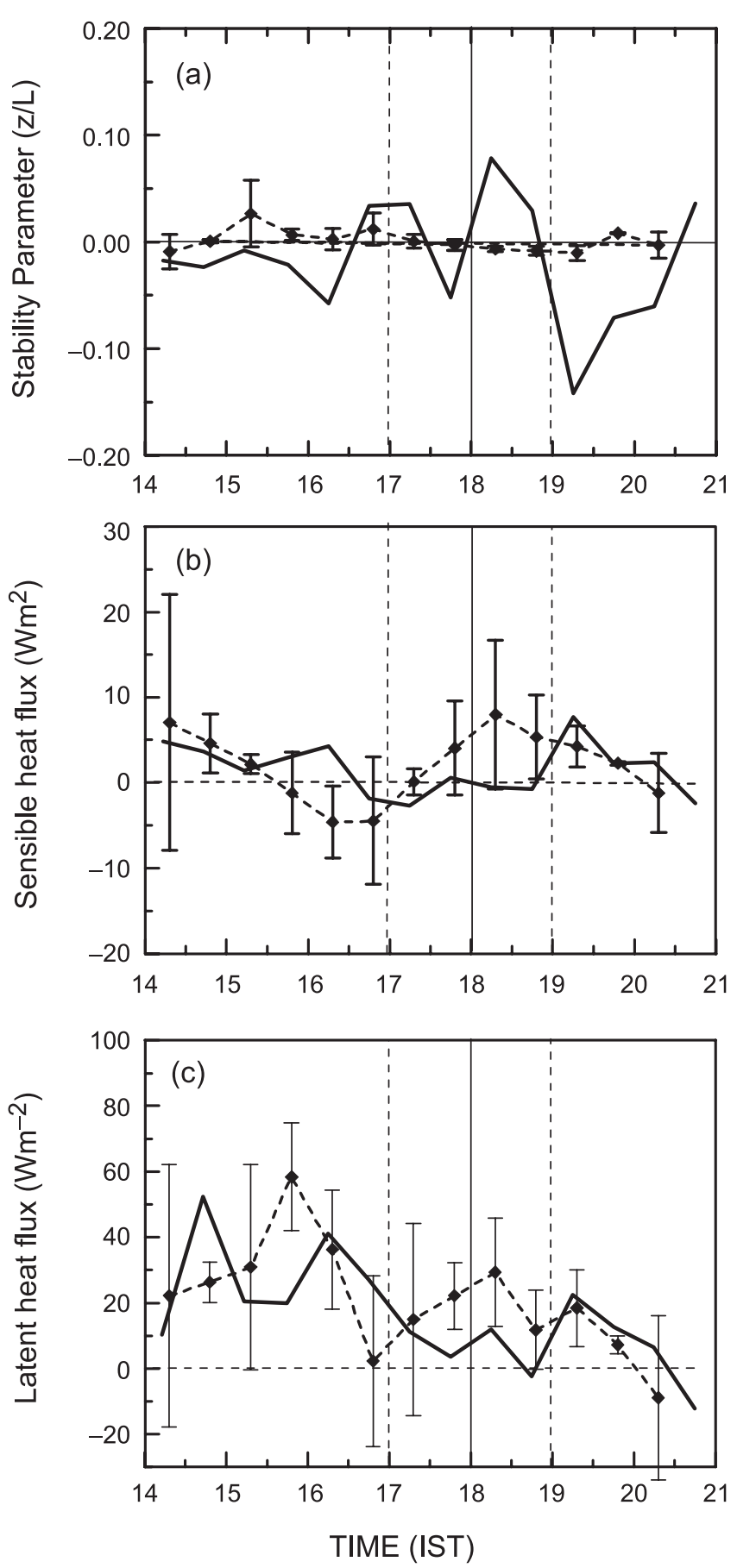

Figure 8. Same as in figure 6 but for (a) stability parameter, (b) sensible heat flux and (c) latent heat flux.

We could not make an estimate of surface energy balance since the net radiation measurements were not made during the experiments.

\section{Discussions and conclusions}

Intensive observations of atmospheric surface layer parameters were carried out during the total solar eclipse of August 11th, 1999 over a semi arid region Ahmedabad, India. The temperature is found to decrease by $\sim 0.5^{\circ} \mathrm{C}$, wind speed is also showing a decrease, while the variation in relative humidity is within the natural variability of the day. The temperature did not regain to the mean value just after the fourth contact (1900 hrs) and sunset was at $1925 \mathrm{hrs}$. It is found that air temperature showed a decrease after the sunset. The soil temperature also showed similar variations in the upper levels of the soil associated with changes in air temperature following the eclipse. This can be associated with the radiative cooling in the ground surface. In addition to this, the radiative divergence within the air can cause cooling in the boundary layer above the ground. Since the solar eclipse in India occurred in the evening hours and partial cloudiness prevailed during the period of observation, the changes in the mean parameters noted are not so drastic as reported earlier. The minimum of wind speed occurred during the solar eclipse close to the totality and followed by an increase in wind speed by the end phase of the eclipse, as reported in earlier studies (Arulraj et al 1998 and Dolas et al 2002). However, Eaton et al (1997) reported natural variability in wind speed following the eclipse over Delinquin, Australia during the solar eclipse of 1994. This indicates that the degree to which each parameter or process is affected depends on the time of the day, latitude, soil conditions, background wind conditions, degree of obscuration, complexity of terrain and type of land use (Eaton et al 1997).

The decrease in wind speed during the eclipse period may be due to the suppression of turbulence and also due to surface cooling and the stabilisation of the surface layer following the drop in temperature. The decrease of the turbulence process is evident from: the decrease in spectral power of wind and temperature spectra, the decrease in the rate of dissipation of TKE, friction velocity, TKE, and also from the sensible and latent heat fluxes during the solar eclipse. The stability parameter provided evidence for the stabilisation following the total phase of the solar eclipse. The suppression of turbulence must be the result of the stabilisation of the surface layer during the solar eclipse.

\section{Acknowledgement}

We thank Dr. A K S Gopalan, Director, Space Application Centre, Ahmedabad for providing all the support for conducting the boundary layer experiments during the period at SAC campus. We are also grateful to Dr. P C Joshi, Head, Atmospheric Science Division, SAC, Ahmedabad for the support and encouragement during the campaign. Our thanks to Sunshine Enterprises, 
Bangalore, for help in the setting up of the experiment during the campaign. We thank the anonymous reviewer for constructive comments that helped improve the manuscript.

\section{References}

Anderson R C, Ikeefer D R and Mayers O E 1972 Atmospheric and temperature changes during 7 March 1970 solar eclipse; J. Atmos. Sol. Terr. Phys., 29 583-587

Antonia R A, Chambers A J, Phong-Anant D , Rajagopalan S and Sreenivasan K R 1979 Response of atmospheric surface layer turbulence to a partial solar eclipse; J. Geophys. Res. 4 1689-1692

Arulraj S, Dharmaraj T, Debaje S B, Badgavanth Singh A and Vernekar K G 1998 The dynamics of the surface layer during total solar eclipse 1995; Mausam 49 475-480

Caughey S J and Wyngaard J C 1979 Turbulent kinetic energy in convective conditions; Q. J. Roy. Meteorol. Soc. $105231-239$

Caughey S J and Palmer S G 1979 The behaviour of the turbulent statistics through the depth of the convective boundary layer and into the stable air of the free atmosphere; Q. J. Roy. Meteorol. Soc. 105 811-827

Chimonas G and Hines C O 1970 Atmospheric gravity waves induced by a solar eclipse; J. Geophys. Res. 75875

Dolas P M, Ramachandran R, Sen Gupta K, Patil S M and Jadhav PN 2002 Atmospheric surface layer processes during the total solar eclipse of 11 August 1999; Boundary Layer. Meteorol. 104 445-461

Eaton F D, Hines J R, Hatch W H, Cionco R M, Byers J and Garvey D 1997 Solar eclipse effects observed in the planetary boundary layer over a desert; Boundary-Layer Meteorol. 83 331-346

Jenkins G M and Watts D G 1968 Spectral analysis and its applications (California: Holden Day Inc)

Kaimal J C and Finnigan J J 1994 Atmospheric Boundary Layer flows, their structure and measurement; (Oxford: Oxford University Press)

Kunhikrishnan P K and Krishna Murthy B V 1982 Atmospheric pressure perturbations during total solar eclipse on 16 February 1980; Proc. Indian Natn. Sci. Acad. 48A Supplement no 33 238-253

Liu J Y, Hsiao C C, Tsai L C, Liu C H, Kuo F S, Lue H Y, Huang C M 1998 Vertical phase and group velocities of internal gravity waves derived from ionograms during the solar eclipse of 24 October 1995; J. Atmos. Sol. Terr. Phys. 60 1679-1686

Narasimha R, Prabhu A, Narahari Rao K and Prasad C R 1982 Atmospheric Boundary Layer Experiment; Proc. of INSA Bull, 48 175-186

Praveena K and P K Kunhikrishnan 2002 Some characteristics of atmospheric surface layer over a tropical inland region during south west monsoon period; Atmos. Research 62 11-124

Sethuraman 1982 Dynamics of the atmospheric boundary layer during the 1980 total solar eclipse; Proc. of INSA Bull 48 187-195

Singh J 1998 Total solar eclipse of August 11 1999; Bull. Astro.Soc. India 26 717-730

Stull R B 1988 An introduction to boundary layer meteorology (Dordrecht: Kluwer Academic Publishers)

Winston J K, Radhika R, Nair K N, Sengupta K and Kunhikrishnan P K 1993 On the spectral behaviour of atmospheric boundary layer parameters at Thumba India; Q. J. Roy. Meteorol. Soc. 119 187-197 